\title{
Absorbed Radiation Dose in Radiosensitive Organs Using 64- and 320-Row Multidetector Computed Tomography: A Comparative Study
}

\author{
Atif N. Khan, ${ }^{1}$ Waqas Shuaib, ${ }^{2}$ Boris Nikolic, ${ }^{3}$ Mohammad K. Khan, ${ }^{2}$ \\ Jian Kang, ${ }^{2}$ and Faisal Khosa ${ }^{1,2}$ \\ ${ }^{1}$ Department of Radiology, Beth Israel Deaconess Medical Center, Harvard Medical School, Boston, MA 02215, USA \\ ${ }^{2}$ Department of Radiology and Imaging Sciences, Emory University Hospital, Atlanta, GA 30308, USA \\ ${ }^{3}$ Department of Radiology, Albert Einstein Medical Center, Philadelphia, PA 19141, USA
}

Correspondence should be addressed to Faisal Khosa; faisal.khosa@emoryhealthcare.org

Received 9 February 2014; Accepted 16 June 2014; Published 6 August 2014

Academic Editor: Albert V. Bruschke

Copyright (c) 2014 Atif N. Khan et al. This is an open access article distributed under the Creative Commons Attribution License, which permits unrestricted use, distribution, and reproduction in any medium, provided the original work is properly cited.

\begin{abstract}
Aim. To determine absorbed radiation dose (ARD) in radiosensitive organs during prospective and full phase dose modulation using ECG-gated MDCTA scanner under 64- and 320-row detector modes. Methods. Female phantom was used to measure organ radiation dose. Five DP-3 radiation detectors were used to measure ARD to lungs, breast, and thyroid using the Aquilion ONE scanner in 64- and 320-row modes using both prospective and dose modulation in full phase acquisition. Five measurements were made using three tube voltages: 100, 120, and $135 \mathrm{kVp}$ at $400 \mathrm{~mA}$ at heart rate (HR) of 60 and $75 \mathrm{bpm}$ for each protocol. Mean acquisition was recorded in milligrays (mGy). Results. Mean ARD was less for 320-row versus 64-row mode for each imaging protocol. Prospective EKG-gated imaging protocol resulted in a statistically lower ARD using 320-row versus 64-row modes for midbreast (6.728 versus $19.687 \mathrm{mGy}, P<0.001)$, lung $(6.102$ versus $21.841 \mathrm{mGy}, P<0.001)$, and thyroid gland $(0.208$ versus $0.913 \mathrm{mGy} ; P<0.001)$. Retrospective imaging using 320- versus 64-row modes showed lower ARD for midbreast (10.839 versus $43.169 \mathrm{mGy}, P<0.001$ ), lung (8.848 versus $47.877 \mathrm{mGy}, P<0.001$ ), and thyroid gland ( 0.057 versus $2.091 \mathrm{mGy} ; P<0.001)$. ARD reduction was observed at lower $\mathrm{kVp}$ and heart rate. Conclusions. Dose reduction to radiosensitive organs is achieved using 320-row compared to 64-row modes for both prospective and retrospective gating, whereas 64-row mode is equivalent to the same model 64-row MDCT scanner.
\end{abstract}

\section{Introduction}

Multidetector computed tomography angiography (MDCTA) is uniquely suited to study cardiac anatomy and coronary artery disease (CAD) in a noninvasive manner and may even provide additional prognostic information to the baseline risk stratification [1]. The high sensitivity (85\%-95\%) and specificities (83-90\%) of the newer generation scanners have already been documented $[2,3]$. The high negative predictive value to rule out coronary artery disease (CAD) has prompted current European Society of Cardiology guidelines on management of stable angina to recommend MDCTA for patients with a low pretest probability of CAD and inconclusive stress testing [4].
The advent of 64- and 320-row MDCT have further improved the volume coverage, $z$-axis resolution, and scanning speed which has resulted in an increase in the number of potential applications of this technology and also the average scanned volume per examination. The new 320-row MDCT also produces better image quality as compared to the current 64-row MDCT [5]. The radiation dose associated with MDCT remains the single most significant concern in the context of its wider acceptability as a screening and diagnostic test $[6,7]$. As MDCT is considered a major source of ionizing radiation in medicine, further in depth study of radiation exposure with these new scanners is therefore paramount $[8,9]$. 


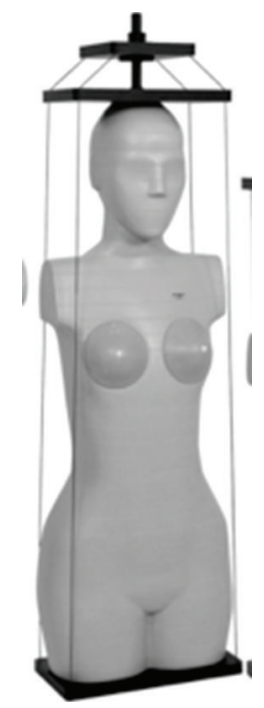

FIgURE 1: CIRS ATOM female phantom (Model number 702-D).

Amongst the various parameters which affect and account for the amount of radiation required during MDCTA examination are maximum body mass index (BMI), tube voltage $(\mathrm{kVp})$, retrospective versus prospective image acquisition, volume scan length, heart rate (HR), and tube current time product (mAs) [10-17]. In addition, the effect of $\mathrm{kVp}$ variation on radiation dose depends on the type of CT scanner due to individualized internal scanner filtration and geometry. Also, results of $\mathrm{kVp}$ variations have generally been evaluated for the computed tomography dose index (CTDI) and/or CTDI water $\left(\mathrm{CTDI}_{\mathrm{w}}\right)$ but not as they pertain to specific organ doses $[10,16]$. Reduction of $\mathrm{kVp}$ from 135 to 120 or 120 to 100 during cardiac imaging has also been shown to produce adequate image quality [5].

The objectives of our study were to measure and compare the ARD in radiosensitive organs (breast, lung, and thyroid) during MDCTA under the 64- and 320-row modes of operation to evaluate the effect of changes in $\mathrm{HR}$ and $\mathrm{kVp}$ on the ARD in these organs. Prior publications have emphasized the use of direct organ detectors for accurate measurement of ARD instead of the Monte Carlo System [18, 19].

\section{Material and Methods}

IRB approval was waived for this experimental study, which was performed on anthropomorphic phantom and did not involve patients or animals. All images were acquired with Toshiba Aquilion ONE MDCT scanner.

2.1. Phantom and Detectors. The CIRS ATOM female phantom (Model number 702-D, Computed Imaging Reference Systems Inc., Norfolk, VA) is an anthropomorphic, cross sectional dosimetry phantom designed to measure organ radiation dose (Figure 1). The ATOM female phantom is manufactured with 38 slabs, $25 \mathrm{~mm}$ in thickness (each). It is important to mention that female phantom was used, specifically, to determine the absorbed radiation dose to

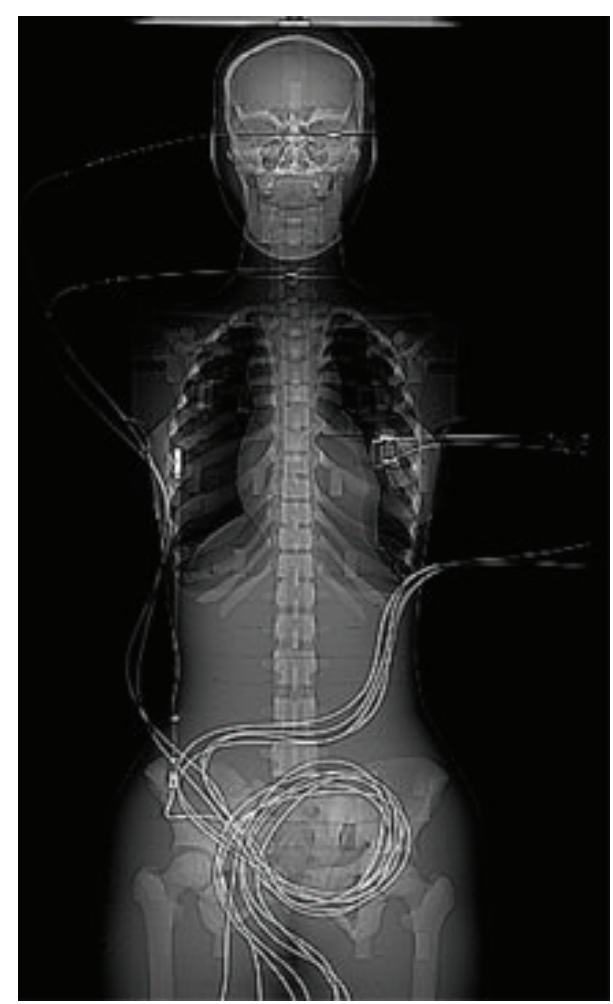

(a)

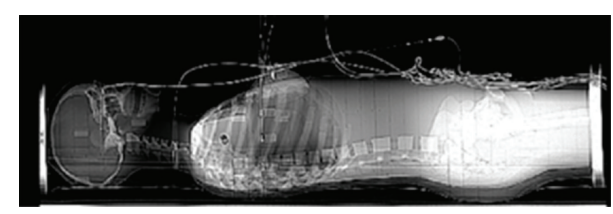

(b)

FIgure 2: Phantom in supine and lateral view with detectors location.

the breast. Apart from the breasts, the female is equivalent to a small male phantom making it the ideal phantom to use. The phantom slabs are tightly bound together when in use for dosimetry measurements (Figure 1) with four strings, resulting in minimal interfaces between the slabs when viewed as a scout (Figure 2). The phantom provides predetermined locations specific to 21 internal body organs. Five custom made DP-3 solid state detectors (RTI Electronics AB, Molndal, Sweden) were used to measure absorbed radiation dose (ARD) to lungs, breast, and thyroid using different scan protocols on the Aquilion ONE scanner. The DP-3 detectors had been previously calibrated by RTI for measurements matched to the primary beam quality of the CT scanners. The protocols employed were designed to quantify radiation doses for prospective gated and dose modulation coronary angiography using MDCT. Three DP-3 detectors were placed in section 16 at locations 72,73 , and 75 representing the anterior, middle, and posterior locations in upper lobe of left lung, respectively. One DP-3 dose detector was placed in the right breast corresponding to the midbreast. One DP-3 detector was placed in section 10, location 26 representing 
the left lobe of the thyroid gland (Figures 2(a) and 2(b)). The output signals from these five detector probes were fed to the Barracuda Electrometer (RTI Electronics) equipped with multiple electrometer modules to accommodate concurrent data collection. The dosimeter information is in turn fed into a laptop computer running "Ocean" Software (RTI Electronics) for display and storage.

2.2. CT Imaging Protocols. All scans were performed with the Aquilion ONE 320-MDCT scanner under two different scan modes (64-row versus 320-row). The Aquilion ONE MDCT scanner is configured to operate as a 320-row MDCT and a 64-row MDCT. Under the 64-row mode of operation, the Aquilion ONE behaves essentially the same as the Aquilion 64-row MDCT. The anthropomorphic phantom was scanned in a single continuous session using both modes for prospective gated and dose modulated coronary MDCTA. The phantom was placed in supine position with phantom head entering the CT gantry first. Scout images were taken in anteroposterior and lateral direction to ensure the same volume coverage so data acquisition could be standardized for all measurements. All data were collected using the same $220 \mathrm{M}$ (medium acquisition FOV) display field of view (DFOV) and $160 \mathrm{~mm}$ volume scan length (VSL). The display field of view (D-FOV) is part of the reconstruction algorithm that sets the display size, pixel size, and display characteristics and volume scan length (VSL) is the area covered by the scanner along the $z$-axis. The mean of five radiation dose measurements for each organ at each $\mathrm{HR}$ and $\mathrm{kVp}$ was converted into the effective organ dose by multiplying it with the appropriate tissue-weighting factor for each organ $[18,19]$.

2.3. Coronary CT Angiography. MDCTA protocols (prospective gating) were studied at three different tube voltages 100,120 , and $135 \mathrm{kVp}$ with a constant $400 \mathrm{~mA}$ tube current simulating two different heart rates at 60 and 75 beats per minute (bpm) with 64-row and 320-row modes. The volume scan length for each scan extended from one centimeter below the carina to the base of the heart.

In 64-row mode, prospectively gated MDCTA technique images heart with a step and shoot helical method with images captured every alternate heart beat. Gantry rotation time was $0.35 \mathrm{msec}$ with $8.1 \mathrm{msec}$ total scan time with a pitch of 17.1. A volume scan length of $160 \mathrm{~mm}$ and DFOV of $220 \mathrm{~mm}$ were used to standardize all measurements. At $75 \mathrm{bpm}$, the Aquilion ONE in 64-row mode operates differently and acquires images of the heart with a dose modulation technique of $100 \mathrm{~mA}$ tube current during 30$90 \%$ of the R-R interval with a peak $\mathrm{mA}$ of 400 at $75 \%$ of the $\mathrm{R}-\mathrm{R}$ interval. The table's pitch changes to 12.1 corresponding to 0.197 with an increased total scan time of $10.4 \mathrm{msec}$. Again, five datasets were taken and recorded for both heart rates using three different tube voltages (average of 5 was taken and recorded as the actual measurement).

The 320-row mode provides two different techniques for cardiac imaging: a prospective CTA and a second complete functional analysis with dose modulation. Under the 320-row mode, a total of 320 rows of detectors are available with a total
TABLE 1: Comparison of Absorbed Radiation Dose (ARD) (mGy) for radiosensitive organs (Lung, Breast and Thyroid) between prospective imaging at 320-row MDCT versus prospective/step \& shoot method at 64-row MDCT adjusting the effects of tube voltages and heart rates.

\begin{tabular}{lccc}
\hline Locations & 320-row mode & 64-row mode & $P$ value \\
\hline Left Lung & 6.102 & 21.841 & $<0.001$ \\
Left Breast & 6.728 & 19.687 & $<0.001$ \\
Thyroid (Left lobe-26) & 0.208 & 0.913 & $<0.001$ \\
\hline
\end{tabular}

collimator width of $(320 \times 0.5 \mathrm{~mm})=160 \mathrm{~mm}$ per rotation for data acquisition enabling the scanner to image the entire heart in a single gantry rotation $(0.35 \mathrm{msec})$. The prospective CTA technique images the heart during $65-85 \%$ of the R-R interval with a slice thickness of $0.5 \mathrm{~mm}$. A tube current of $400 \mathrm{~mA}$, D-FOV of $220 \mathrm{~mm}$ medium, and VSL of $160 \mathrm{~mm}$ was used to take all measurements while utilizing three different tube voltages $(100,120$, and $135 \mathrm{kVp})$ at two different heart rates ( 60 and $75 \mathrm{bpm})$.

The 64-row mode automatically selects modulation technique at a higher heart rate of $75 \mathrm{bpm}$. Tube modulation technique was also studied using the 320-row mode. The basic concept is almost the same with a baseline current of $100 \mathrm{~mA}$ during a complete R-R interval with a peak $\mathrm{mA}$ of 400 at $75 \%$ of the R-R interval. The CTA protocol with three different tube voltages at both heart rates was measured and recorded.

2.4. Statistical Analysis. Two-sample $t$ tests were performed to detect the difference in ARD at breast, lung, and thyroid between the 320-row MDCT and the 64-row MDCT for each of the imaging protocols. In a subanalysis, linear mixed effects models were fitted to study both effects of tube voltage and heart rate variation on ARD, respectively, while adjusting for all other imaging factors (imaging protocols, scanners, etc.). In each model, a random intercept is specified to model random effects for multiple measurements. Wald tests have been used to determine the significance of effects for each factor.

\section{Results}

Mean ARD at 320-row versus 64-row modes were: midbreast (6.728 versus $19.687 \mathrm{mGy}, P<0.001$ ), lung (6.102 versus $21.841 \mathrm{mGy}, P<0.001)$, and thyroid gland $(0.208$ versus 0.913 mGy; $P<0.001$ ) (Table 1). Similarly with the dose modulation protocol, the ARD in the organs for the 320-row versus 64 -row modes were as follows: midbreast (10.839 versus $43.169 \mathrm{mGy}, P<0.001$ ), lung (8.848 versus $47.877 \mathrm{mGy}$, $P<0.001)$, and thyroid gland $(0.057$ versus $2.091 \mathrm{mGy} ; P<$ 0.001 ) (Table 3). The 320-row mode showed a significantly lower ARD at all detector's locations when compared to the 64-row mode equivalent for both imaging protocols.

In a subanalysis, linear mixed effects models were fitted to study the effects of tube voltage and heart rate variation on ARD while adjusting for all other imaging factors (Tables 2 and 4). In our study, the reduction of $\mathrm{kVp}$ from 135 to $100 \mathrm{kVp}$ for prospective ECG gated MDCTA performed on 
TABLE 2: The effect of tube voltage on ARD (mGy) for prospective EKG gated/step \& shoot method at both 64-row and 320-row protocols.

\begin{tabular}{lccc}
\hline Protocols & $\mathrm{kVp} \mathrm{100}$ & $\mathrm{kVp} \mathrm{120}$ & $P$ value \\
\hline 320-row mode & 10.98 & 17.21 & 0.087 \\
64-row mode & 14.47 & 22.30 & 0.147 \\
\hline Protocols & $\mathrm{kVp} \mathrm{100}$ & $\mathrm{kVp} \mathrm{135}$ & $P$ value \\
\hline 320-row mode & 10.98 & 22.09 & $<0.05$ \\
64-row mode & 14.47 & 29.97 & $<0.05$ \\
\hline
\end{tabular}

TABLE 3: Comparison of ARD (mGy) for radiosensitive organs (Lung, Breast and Thyroid) between dose modulation imaging at 320-row MDCT versus dose modulation method at 64-row MDCT.

\begin{tabular}{lccc}
\hline Locations & 320-row scanner & 64-row scanner & $P$ value \\
\hline Left Lung & 8.848 & 47.88 & $<0.001$ \\
Left Breast & 10.84 & 43.17 & $<0.001$ \\
Thyroid (Left lobe-26) & 0.057 & 2.091 & $<0.001$ \\
\hline
\end{tabular}

TABLE 4: The effect of tube voltage variation on ARD ( $m G y)$ for dose modulation protocol at both 64-row and 320-row modes.

\begin{tabular}{lccc}
\hline Protocols & $\mathrm{kVp} \mathrm{100}$ & $\mathrm{kVp} \mathrm{120}$ & $P$ value \\
\hline 320-row mode & 17.57 & 27.15 & 0.072 \\
64-row mode & 38.76 & 61.34 & 0.131 \\
\hline Protocols & $\mathrm{kVp} \mathrm{100}$ & $\mathrm{kVp} \mathrm{135}$ & $P$ value \\
\hline 320-row mode & 17.574 & 34.26 & $<0.05$ \\
64-row mode & 38.76 & 80.61 & $<0.05$ \\
\hline
\end{tabular}

TABLE 5: The effect of heart rate variation on the absorbed radiation dose (mGy) for both scanners.

\begin{tabular}{lccc}
\hline Modes & $60 \mathrm{bpm}$ & $75 \mathrm{bpm}$ & $P$-value \\
\hline 320-row volume mode & 15.50 & 27.59 & $<0.001$ \\
64-row helical mode & 22.25 & 60.24 & $<0.001$ \\
\hline
\end{tabular}

64-row mode and 320-row mode demonstrated a significant radiation dose reduction of $51.72 \%$ and $50.2 \%$, respectively. However, it was noticed that a similar significant effect was not observed with a 120 to $100 \mathrm{kVp}$ reduction in tube voltage. Similarly, the influence of variation in heart rate on ARD using prospective imaging methods was studied (Table 5). A similar trend was seen with dose modulation imaging for 64row versus 320 -row modes showing a reduction of $51.92 \%$ and $48.72 \%$ in ARD, respectively (Table 6). At a lower heart rate of $65 \mathrm{bpm}$, the 64 -row mode delivered $63.1 \%$ less radiation dose while the 320-row mode delivered $43.82 \%$ less dose compared to doses delivered by each mode at $75 \mathrm{bpm}$.

\section{Discussion}

In our study, we measured the $\mathrm{ARD}$ with prospective gating as it delivers the lowest dose for assessment of coronary artery disease; similarly, we used DP3 detectors for acquisition of ARD measurements which are characterized by increased ease of use compared to thermoluminescent dosimeters (TLD) - the prior gold standard for point radiation dose measurement. In our study, ARD was measured for the most radiosensitive organs that are within the primary radiation beam (lung and breast). A small amount of osseous material (bone marrow) does lie within the primary beam and is, therefore, subjected to some degree of stochastic radiation. Thus, ARD in the sternum is well approximated by the chest wall measurement. Other organs within the primary beam such as neural, cardiac, and esophageal tissue are relatively radioresistant. Additional dose measurements addressing these anatomic structures were, therefore, not conducted in this study.

The differences seen above indicate a higher ARD with 64-row mode in radiosensitive organs in the scan field and attests to a greater radiation safety profile for the 320-row mode as studied on a standardized phantom. Our study supports the use of tube voltage and heart rate reduction to decrease ARD and is in synchrony with Matsubara et al. who obtained similar results on phantom studies with 64-row CT angiography [18]. Litmanovich et al. [20] also found better ARD profile in 64-row MDCT phantom study by using imaging protocols with lower tube voltage which was in consensus with an earlier study by Hurwitz et al. $[21,22]$. Our study adds to the literature by documenting similar trend of $\mathrm{kVp}$ and $\mathrm{HR}$ variation on $\mathrm{ARD}$ seen in radiosensitive organs when using 320-row mode. Also, it advocates better radiation reduction of the 320-row mode for these radiosensitive organs when compared to the 64row mode. Again, 64-row mode is similar to 64-row MDCT scanner in functionality.

Our data along with those of others also indicate that occurrence of deterministic skin effects secondary to MDCTA with the dual source 64- and 320-detector-row modes is fundamentally inconceivable, even in the setting of performance of multiple studies in a short time span in the same individual as deterministic effects have a threshold between 2,000 and 20,000 mGy, depending on the severity of the radiation damage.

Limitations. Our study has a few limitations. First, only one body type anthropomorphic phantom was used. Patients with higher BMIs may receive ARDS that deviate from our results due to increased scatter radiation and the necessity to potentially increase tube current and voltage to obtain adequate image quality. ARD measured in lung and breast is a local dose and does not represent the equivalent organ dose.

The actual doses for any individual will vary from patient to patient, depending on tube current $(\mathrm{mA})$ setting, heart rate, $z$-axis coverage, and body habitus. For prospective gating used in this study, absorbed organ doses are linearly proportional to the $\mathrm{mA}$ levels. This allows for easy calculations of different $\mathrm{mA}$ levels and makes additional radiation dose measurements unnecessary. Other scanning protocols, on the other hand, such as tube modulation would have a more complex effect on ARD and necessitate additional measurements. Finally, there is a lack of radiation data correlation to image quality or diagnostic accuracy assessment. The aim of this paper was to assess ARD through 
TABLE 6: Percentage reduction in ARD with 320-row compared to 64-row mode.

\begin{tabular}{lcc}
\hline Detector site & $\begin{array}{c}\text { Percentage reduction in ARD (mGy) with 320 row mode MDCTA compared to 64-row mode MDCTA } \\
\text { Prospective ECG-gating protocol }\end{array}$ \\
\hline Left Lung & $72 \%$ & $81.52 \%$ \\
Left Mid-Breast & $65.8 \%$ & $74.8 \%$ \\
Breast Surface & $64.53 \%$ & $78.3 \%$ \\
Thyroid & $77.2 \%$ & $97.2 \%$ \\
\hline
\end{tabular}

the comparison of 64-row and 320-row MDCTs as well as differing voltages. Because the anthropomorphic phantoms do not have actual cardiopulmonary structures, only the ARD could be measured while image quality cannot be assessed.

\section{Conclusion}

Coronary CT angiography can be performed with 320row mode with much less $A R D$ to radiosensitive organs as compared to 64-row mode at Aquilion ONE. Use of appropriate tube voltage $(\mathrm{kVp})$ and heart rate control can further reduce the radiation dose to radiosensitive organs.

\section{Disclosure}

Faisal Khosa is the (2013-2015) American Roentgen Ray Scholar.

\section{Conflict of Interests}

The authors declare that there is no conflict of interests regarding the publication of this paper. There are no financial, consultant, institutional, and other relationships that might lead to bias or a conflict of interests.

\section{References}

[1] E. Choi, S. I. Choi, J. J. Rivera et al., "Coronary computed tomography angiography as a screening tool for the detection of occult coronary artery disease in asymptomatic individuals," Journal of the American College of Cardiology, vol. 52, no. 5, pp. 357-365, 2008.

[2] J. M. Miller, C. E. Rochitte, M. Dewey et al., "Diagnostic performance of coronary angiography by 64-row CT," The New England Journal of Medicine, vol. 359, no. 22, pp. 2324-2336, 2008.

[3] M. J. Budoff, D. Dowe, J. G. Jollis et al., "Diagnostic performance of 64-multidetector row coronary computed tomographic angiography for evaluation of coronary artery stenosis in individuals without known coronary artery disease: results from the prospective multicenter ACCURACY (Assessment by Coronary Computed Tomographic Angiography of Individuals Undergoing Invasive Coronary Angiography) trial," Journal of the American College of Cardiology, vol. 52, no. 21, pp. 1724-1732, 2008.

[4] K. Fox, M. Á. A. García, D. Ardissino et al., "Guidelines on the management of stable angina pectoris: executive summary," Revista Espanola de Cardiologia, vol. 59, no. 9, pp. 919-970, 2006.
[5] H. Matsutani, T. Sano, T. Kondo et al., "Comparison of radiation dose reduction of prospective ECG-gated one beat scan using 320 area detector CT coronary angiography and prospective ECG-gated helical scan with high helical pitch (FlashScan) using 64 multidetector-row CT coronary angiography," Nippon Hoshasen Gijutsu Gakkai Zasshi, vol. 66, no. 12, pp. 1548-1554, 2010.

[6] D. J. Brenner and H. Hricak, "Radiation exposure from medical imaging: time to regulate?" Journal of the American Medical Association, vol. 304, no. 2, pp. 208-209, 2010.

[7] D. J. Brenner and E. J. Hall, "Computed tomography: an increasing source of radiation exposure," New England Journal of Medicine, vol. 357, no. 22, pp. 2277-2284, 2007.

[8] S. Achenbach, "Computed tomography coronary angiography," Journal of the American College of Cardiology, vol. 48, no. 10, pp. 1919-1928, 2006.

[9] A. J. Einstein, "Radiation risk from coronary artery disease imaging: how do different diagnostic tests compare?" Heart, vol. 94, no. 12, pp. 1519-1521, 2008.

[10] J. Hausleiter, T. Meyer, M. Hadamitzky et al., "Radiation dose estimates from cardiac multislice computed tomography in daily practice: impact of different scanning protocols on effective dose estimates," Circulation, vol. 113, no. 10, pp. 1305$1310,2006$.

[11] A. Gopal, S. S. Mao, D. Karlsberg et al., "Radiation reduction with prospective ECG-triggering acquisition using 64multidetector computed tomographic angiography," International Journal of Cardiovascular Imaging, vol. 25, no. 4, pp. 405416, 2009.

[12] T. C. Gerber, J. Jeffrey Carr, A. E. Arai et al., "Ionizing radiation in cardiac imaging: a science advisory from the American Heart Association Committee on cardiac imaging of the council on clinical cardiology and committee on cardiovascular imaging and intervention of the council on cardiovascular radiology and intervention," Circulation, vol. 119, no. 7, pp. 1056-1065, 2009.

[13] J. Horiguchi, M. Kiguchi, C. Fujioka et al., "Radiation dose, image quality, stenosis measurement, and CT densitometry using ECG-triggered coronary 64-MDCT angiography: a phantom study," American Journal of Roentgenology, vol. 190, no. 2, pp. 315-320, 2008.

[14] A. Khan, K. Nasir, F. Khosa, A. Saghir, S. Sarwar, and M. E. Clouse, "Prospective gating with 320-MDCT angiography: effect of volume scan length on radiation dose," The American Journal of Roentgenology, vol. 196, no. 2, pp. 407-411, 2011.

[15] T. F. Jakobs, C. R. Becker, B. Ohnesorge et al., "Multislice helical $\mathrm{CT}$ of the heart with retrospective ECG gating: reduction of radiation exposure by ECG-controlled tube current modulation," European Radiology, vol. 12, no. 5, pp. 1081-1086, 2002.

[16] M. J. Siegel, B. Schmidt, D. Bradley, C. Suess, and C. Hildebolt, "Radiation dose and image quality in pediatric CT: effect of technical factors and phantom size and shape," Radiology, vol. 233, no. 2, pp. 515-522, 2004. 
[17] M. D. Shapiro, A. J. Pena, J. H. Nichols et al., "Efficacy of prescan beta-blockade and impact of heart rate on image quality in patients undergoing coronary multidetector computed tomography angiography," European Journal of Radiology, vol. 66, no. 1, pp. 37-41, 2008.

[18] K. Matsubara, K. Koshida, K. Noto et al., "Estimation of organabsorbed radiation doses during 64-detector CT coronary angiography using different acquisition techniques and heart rates: a phantom study," Acta Radiologica, vol. 52, no. 6, pp. 632637, 2011.

[19] B. Nikolic, F. Khosa, P. J. Lin et al., "Absorbed radiation dose in radiosensitive organs during coronary $\mathrm{CT}$ angiography using 320-MDCT: effect of maximum tube voltage and heart rate variations," American Journal of Roentgenology, vol. 195, no. 6, pp. 1347-1354, 2010.

[20] D. Litmanovich, D. Tack, P. P. Lin, P. M. Boiselle, V. Raptopoulos, and A. A. Bankier, "Female breast, lung, and pelvic organ radiation from dose-reduced 64-MDCT thoracic examination protocols: a phantom study," The American Journal of Roentgenology, vol. 197, no. 4, pp. 929-934, 2011.

[21] L. M. Hurwitz, T. T. Yoshizumi, P. C. Goodman et al., "Radiation dose savings for adult pulmonary embolus 64-MDCT using bismuth breast shields, lower peak kilovoltage, and automatic tube current modulation," The American Journal of Roentgenology, vol. 192, no. 1, pp. 244-253, 2009.

[22] L. M. Hurwitz, T. T. Yoshizumi, R. E. Reiman et al., "Radiation dose to the female breast from 16-MDCT body protocols," American Journal of Roentgenology, vol. 186, no. 6, pp. 1718-1722, 2006. 


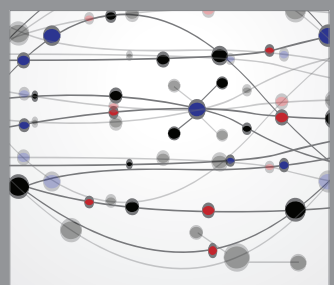

The Scientific World Journal
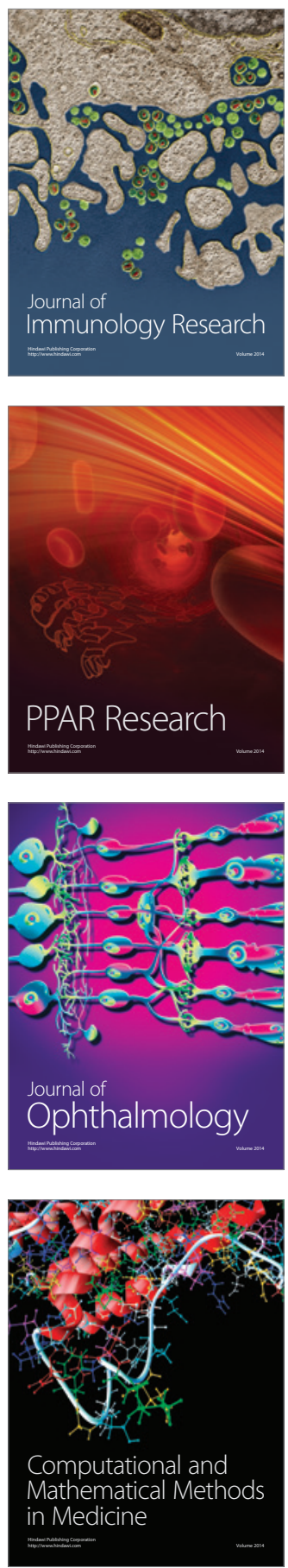

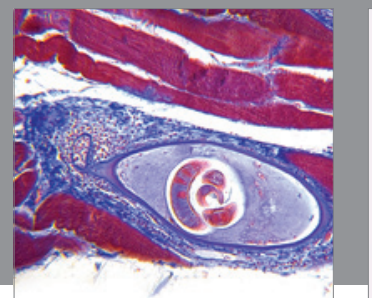

Gastroenterology

Research and Practice
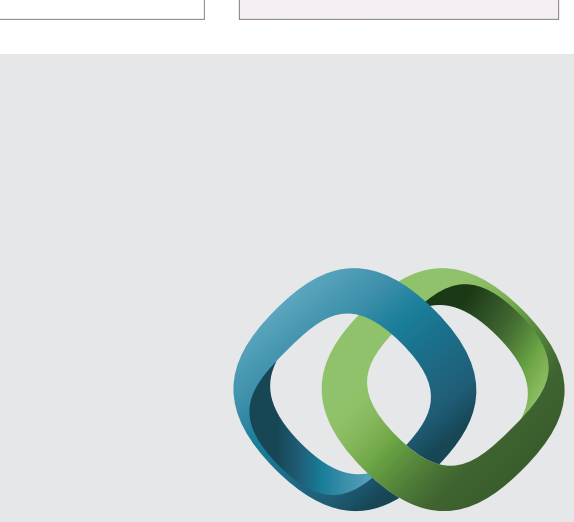

\section{Hindawi}

Submit your manuscripts at

http://www.hindawi.com
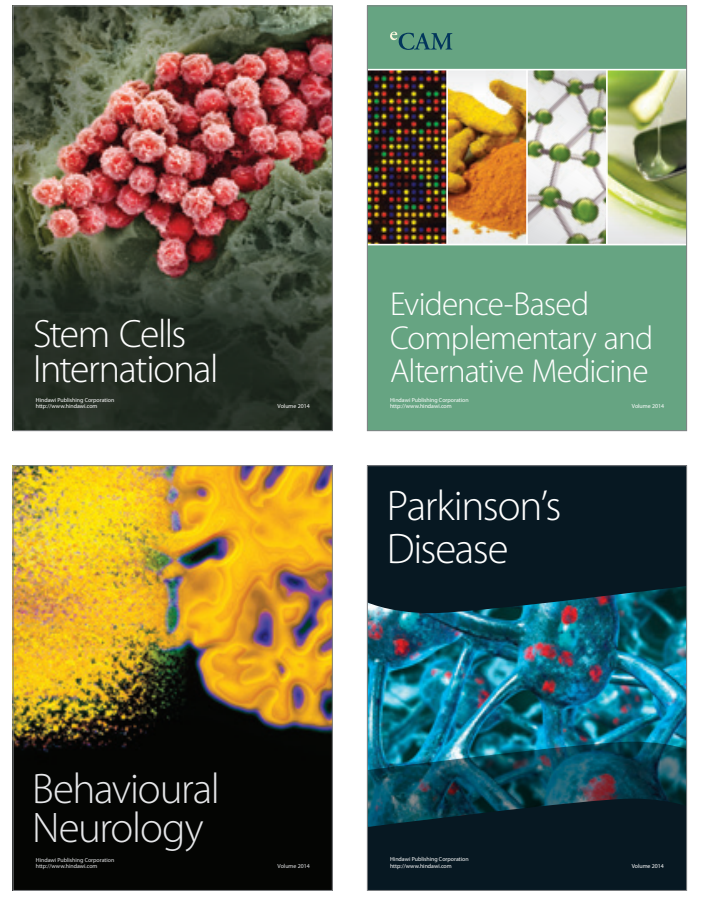
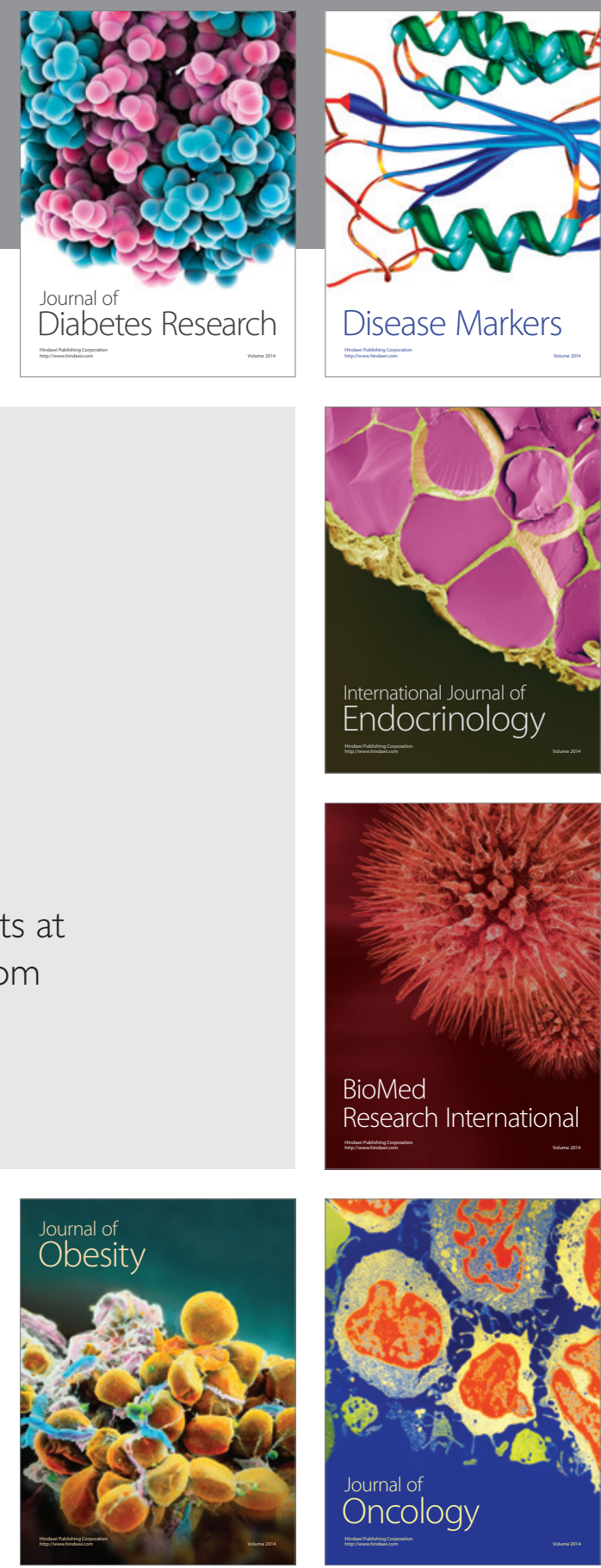

Disease Markers
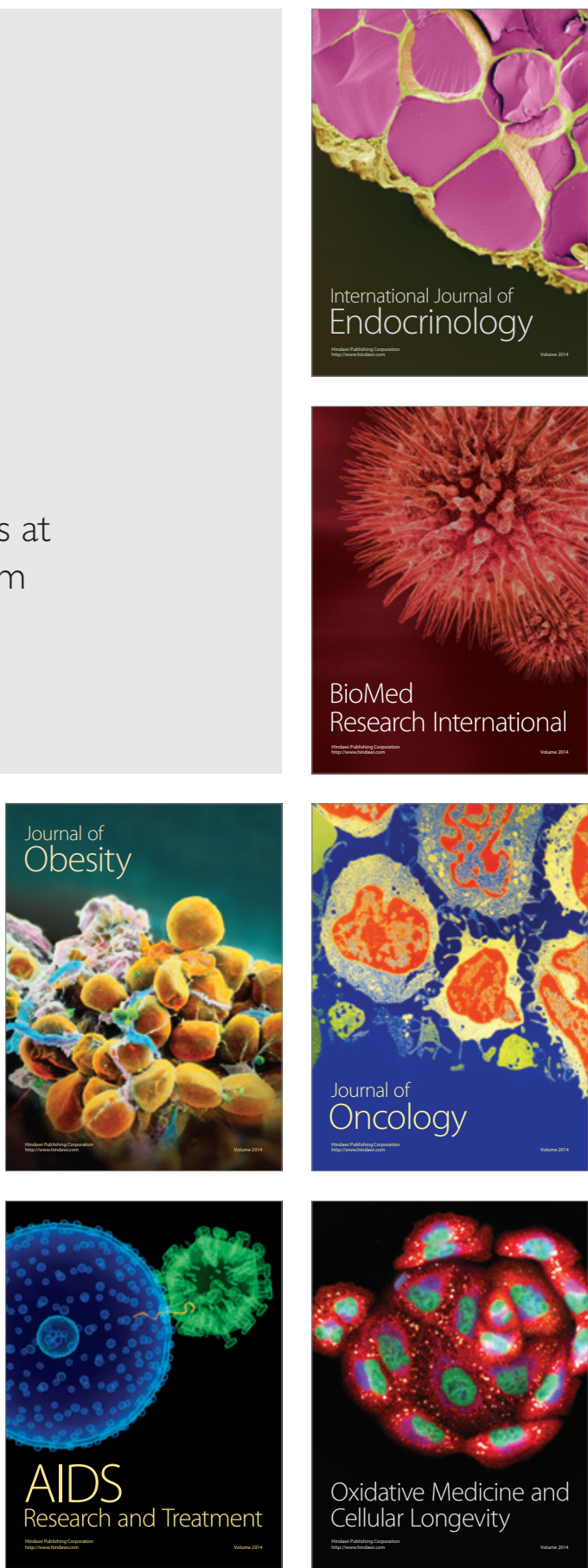